\title{
FREE ACTIONS OF ZERO-DIMENSIONAL COMPACT GROUPS ON MENGER MANIFOLDS
}

\author{
KATSURO SAKAI
}

(Communicated by James West)

\begin{abstract}
It is proved that every separable zero-dimensional compact group acts freely on any Menger manifold $M$. In case $M$ is compact, this result was proved by Dranishnikov. Here is provided an alternative short proof.
\end{abstract}

A manifold modeled on the $n$-dimensional universal Menger compactum $\mu^{n}$ is called a $\mu^{n}$-manifold. In [Dr], Dranishnikov established the following theorem in case $M$ is compact. Here we generalize his theorem in case $M$ is noncompact and provide an alternative short proof.

Theorem. Every separable zero-dimensional compact group ${ }^{1} G$ acts freely on any $\mu^{n}$-manifold $M$.

For a finite group $G$ and $n \in \mathbb{N}$, we define the $n$-dimensional $(n-1)$ connected simplicial complex $L_{G}^{n}$ as the join of $n+1$ copies of $G$ and a free $G$-action on $\left|L_{G}^{n}\right|$ as follows: ${ }^{2}$

$$
g\left(t_{1} g_{1} \oplus \cdots \oplus t_{n+1} g_{n+1}\right)=t_{1} g g_{1} \oplus \cdots \oplus t_{n+1} g g_{n+1} .
$$

Note that each $g \in G$ induces the simplicial isomorphism of $L_{G}^{n}$ onto itself.

Proof of Theorem. By a well-known theorem of Pontryagin [Po, $\S 46, C], G$ is the inverse limit of an inverse sequence of finite groups $G_{i}, i \in \mathbb{N}$; whence $G$ is a subgroup of $\prod_{i \in \mathbb{N}} G_{i}$. We may assume that each $G_{i}$ is nontrivial.

By [Be, the analogue of 5.1.3 (p. 103)], we have a locally finite simplicial complex $K_{0}$ with $\operatorname{dim} K_{0} \leqslant n$ and a proper map $f:\left|K_{0}\right| \rightarrow M$ which induces isomorphisms of homotopy groups of $\operatorname{dim}<n$ and of homotopy groups of ends of $\operatorname{dim}<n$. For each $i \in \mathbb{N}$, we have a nondegenerate $n$-dimensional $(n-1)$-connected finite simplicial complex $L_{i}=L_{G_{i}}^{n}$. We inductively define the simplicial complex $K_{i}$ as the $n$-skeleton of $K_{i-1} \times L_{i}$, where $K \times L$ is the

Received by the editors November 2, 1993.

1991 Mathematics Subject Classification. Primary 57S10, 22C05, 54F15, 54H15.

Key words and phrases. Menger manifold, free action, 0-dimensional compact group.

${ }^{1}$ Topological groups are assumed to be $T_{1}$. Then separable compact groups are metrizable.

${ }^{2}\left|L_{G}^{n}\right|$ is the quotient space of the product space $G^{n+1} \times \Delta^{n}$, where

$$
\Delta^{n}=\left\{\left(t_{1}, \cdots, t_{n+1}\right) \in \mathbf{I}^{n+1} \mid t_{1}+\cdots+t_{n+1}=1\right\},
$$

and the equivalence class of $\left(g_{1}, \cdots, g_{n+1} ; t_{1}, \cdots, t_{n+1}\right)$ is denoted by $t_{1} g_{1} \oplus \cdots \oplus t_{n+1} g_{n+1}$. 
simplicial complex defined by the barycentric subdivision of the cell complex $\{\sigma \times \tau \mid \sigma \in K, \tau \in L\}$. Let $p_{i}:\left|K_{i}\right| \rightarrow\left|K_{i-1}\right|$ be the restriction of the projection. Thus we have an inverse sequence

$$
\left|K_{0}\right| \stackrel{p_{1}}{\longleftarrow}\left|K_{1}\right| \stackrel{p_{2}}{\longleftarrow}\left|K_{2}\right| \stackrel{p_{3}}{\longleftarrow} \cdots .
$$

By using the simplicial approximation theorem and the homotopy extension technique, it is easy to show that if a map $f: \mathbf{S}^{k} \rightarrow\left|K_{i}\right|(k<n)$ extends to a map $f^{\prime}: \mathbf{B}^{k+1} \rightarrow\left|K_{i-1} \times L_{i}\right|$, then $f$ extends to a map $f^{\prime \prime}: \mathbf{B}^{k+1} \rightarrow\left|K_{i}\right|$ such that each $f^{\prime}(x)$ and $f^{\prime \prime}(x)$ are contained in the same simplex in $K_{i-1} \times L_{i}$. By modifying [GHW, Theorem 1], ${ }^{3}$ we can show similarly as [GHW, Theorem 2] that the inverse limit $X$ is a $\mu^{n}$-manifold and that the projection $p_{0}^{\infty}: X \rightarrow\left|K_{0}\right|$ induces the isomorphisms of homotopy groups of $\operatorname{dim}<n$ and of homotopy groups of ends of dim $<n .{ }^{4}$ Since $X$ is homeomorphic to $M$ by [Be, Chapter V, Theorem], we can regard $M=X=\bigcap_{i \in \mathbb{N}}\left(\left|K_{i}\right| \times \prod_{j>i}\left|L_{j}\right|\right) \subset\left|K_{0}\right| \times \prod_{i \in \mathbb{N}}\left|L_{i}\right|$.

Using the free $G_{i}$-action on $\left|L_{i}\right|$, we define a free $G$-action on $\left|K_{0}\right| \times \prod_{i \in \mathbb{N}}\left|L_{i}\right|$ as follows:

$$
\begin{aligned}
& g x=\left(x_{0} ; g_{1} x_{1}, g_{2} x_{2}, \cdots\right) \\
& \quad \text { for } \forall g=\left(g_{1}, g_{2}, \cdots\right) \in G \text { and } \forall x=\left(x_{0} ; x_{1}, x_{2}, \cdots\right) \in\left|K_{0}\right| \times \prod_{i \in \mathbb{N}}\left|L_{i}\right| .
\end{aligned}
$$

Since each $g_{i} \in G_{i}$ induces the simplicial isomorphism of $L_{i}$ onto itself, it is easily observed that $g\left(\left|K_{i}\right| \times \prod_{j>i}\left|L_{j}\right|\right)=\left|K_{i}\right| \times \prod_{j>i}\left|L_{j}\right|$ for each $i \in \mathbb{N}$, which implies $g X=X$. Thus we have a free $G$-action on $M$ as the restriction of the above $G$-action on $\left|K_{0}\right| \times \prod_{i \in \mathbb{N}}\left|L_{i}\right|$.

\section{ACKNOWLEDGMENT}

The author would like to express his thanks to Y. Iwamoto and K. Kawamura for giving helpful information about Menger manifolds.

\section{REFERENCES}

[Be] M. Bestvina, Characterizing k-dimensional universal Menger compacta, Mem. Amer. Math. Soc., vol. 71, Amer. Math. Soc., Providence, RI, 1988.

[Dr] A. N. Dranishnikov, On free actions of zero-dimensional compact groups, Izv. Akad. Nauk SSSR Ser. Mat. 52 (1988), 212-228; English transl., Math. USSR-Izv 32 (1989), 217-232.

[GHW] D. J. Garity, J. P. Henderson, and D. G. Wright, Menger spaces and inverse limits, Pacific J. Math. 131 (1988), 249-259.

[Po] L. S. Pontryagin, Continuous groups, 4th ed., Nauka, Moscow, 1984; English transl., Topological groups, 2nd ed., Gordon and Breach, New York, 1966.

Institute of Mathematics, University of TsukUba, TsukUba-City 305, Japan

E-mail address: sakaiktr@sakura.cc.tsukuba.ac.jp

\footnotetext{
${ }^{3}$ In Theorem 1 of [GHW], if each $X_{i}$ is locally compact and each $p_{i}$ is proper, then $X$ is a $\mu^{n}$-manifold without the condition 2 and it follows from the proof of $(n-1)$-connectedness that the projection of $X$ onto $X_{1}$ induces the monomorphisms of homotopy groups of $\operatorname{dim}<n$ and of homotopy groups of ends of $\operatorname{dim}<n$. Given a complete metric $d_{i}$ for each $X_{i}$ so that $d_{i-1}\left(p_{i}(x), p_{i}(y)\right) \leqslant d_{i}(x, y)$ for each $x, y \in X_{i}$, it suffices to say in the condition 3 that $p_{i+1} h$ is $\varepsilon_{i+1}$-close to $f$, where $\varepsilon_{i}>0(i \in \mathbb{N})$ are prechosen so that $\sum_{i=1}^{\infty} \varepsilon_{i}(=c)<\infty$.

${ }^{4}$ Given $a=\left(a_{1}, a_{2}, \cdots\right) \in \prod_{i \in \mathrm{N}}\left|L_{i}\right|$, we have the embedding $i_{a}:\left|K_{0}\right| \rightarrow M$ defined by $i_{a}(x)=\left(x ; a_{1}, a_{2}, \cdots\right)$. Since $p_{0}^{\infty} i_{a}=\mathrm{id}, p_{0}^{\infty}$ induces the epimorphisms of homotopy groups and of homotopy groups of ends.
} 